\title{
INDUCTION, PAR LE CHLORURE DE TÉTRAZOLIUM, DE LA MUTATION "PETITE COLONIE" CHEZ LA LEVURE
}

\author{
WOLFGANG LASKOWSKI * \\ Laboratoire de Génétique de la Faculté des Sciences \\ Laboratoire de Génétique physiologique du Centre National de la Recherche Scientifique, \\ Paris
}

Received I5.vi.53

\section{INTRODUCTION}

SELON Raut (1953) les mutants à déficience respiratoire de la levure ("petite colonie", Ephrussi, Hottinguer et Chimenes, 1949) proliférant sur milieu solide additionné de sels de tétrazolium, forment des colonies blanches, alors que les colonies formées dans les mêmes conditions par une levure normale sont roses.

$\mathrm{Au}$ cours d'expériences destinées à mettre au point une technique de diagnostic différentiel des deux types de levure, fondée sur l'observation de Raut, Ephrussi (communication verbale) a observé que le chlorure de tétrazolium inhibe électivement la croissance des levures normales et augmente la proportion de colonies de taille réduite. Les caractéristiques biochimiques des cellules constituant ces " petites colonies" sont apparemment identiques à celles des mutants à déficience respiratoire dont la formation spontanée et l'induction par les acridines ont été décrites par Ephrussi et ses collaborateurs. L'augmentation de la proportion de mutants dans les populations proliférant en présence de tétrazolium pouvait, a priori, être due soit à une action sélective, soit à une action mutagène de cette substance. C'est afin d'en éclaircir le mode d'action que nous avons entrepris les expériences décrites ci-dessous.

\section{MATERIEL ET TECHNIQUES}

Toutes les expériences sont effectuées sur la levure de boulangerie (Saccharomyces cerevisie), souche B II (diploïde).

Les cultures en milieu liquide sont faites dans des fioles d'Erlenmayer contenant $25 \mathrm{~cm}^{3}$ d'eau de touraillons (Ephrussi, Hottinguer et Chimènes, 1949) agitées à $25^{\circ}$. L'inoculum comprend environ 5000 cellules.

L'observation des colonies est faite à la suite d'étalements des cultures sur le milieu : Yeast extract, 0,5 p. cent. ; glucose, 3 p. cent. ; gélose, 2 p. cent., en boites Petri et incubation à $25^{\circ}$.

Le même milieu, dépourvu de gélose, est utilisé pour l'isolement des cellules de levure en goutte pendante dans la chambre humide de Winge, à l'aide du micromanipulateur de Fonbrune. La culture de ces cellules a lieu à $25^{\circ}$ également.

* Travail effectué grâce à une bourse du Gouvernement Français. 
Le sel de tétrazolium (TZ) utilisé est le chlorure de 2.3.5. triphényltétrazolium fourni par Paul Lewis Laboratories, Milwaukee, Wisconsin,

$$
\mathrm{R}-\mathrm{C}_{\mathrm{N}-\mathrm{N}^{+}-\mathrm{R}^{\prime}}^{\mathrm{N}-\mathrm{N}-\mathrm{N}-\mathrm{R}^{\prime}} ; \mathrm{R}=\mathrm{R}^{\prime}=\mathrm{R}^{\prime \prime}=\mathrm{C}_{6} \mathrm{H}_{5}-
$$

Une solution aqueuse à $1 \mathrm{p}$. cent. est stérilisée par filtration et conservée au froid et à l'obscurité. Au cours des expériences, les solutions sont exposées le moins longtemps possible à la lumière.

Les concentrations du TZ seront exprimées en gr. par litre.

\section{RESULTATS ET CONCLUSIONS}

Des suspensions de levures sont étalées sur milieu gélosé additionné de diverses quantités de TZ. Pour les concentrations les plus fortes $\left(\mathrm{I} \times 10^{-3}\right.$ et $\left.5 \times 10^{-4}\right)$, on ne décèle à l'oeil nu aucune croissance,

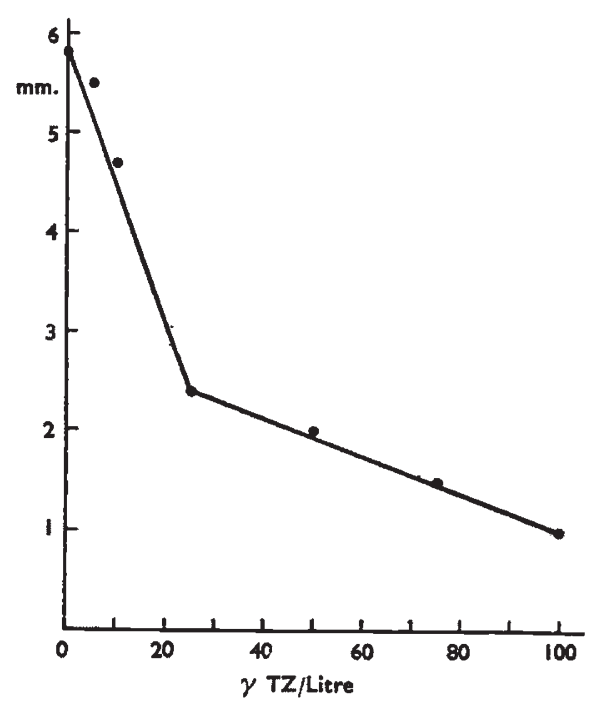

Fic. 1.-Diamètre des colonies (moyennes de 20 déterminations) en fonction de la concentration $\left(\gamma / \mathbf{r}_{\text {. }}\right)$ du milieu en TZ.

même au bout de 5 jours d'incubation. Par contre, dans les cultures témoins, dépourvues de $\mathrm{TZ}$, on observe des colonies au bout de 24 heures; il en est de même lorsque la concentration du milieu en TZ est de $\mathrm{I} \times \mathrm{IO}^{-6}, 5 \times \mathrm{IO}^{-6}$ ou $\mathrm{I} \times \mathrm{IO}^{-5}$. Pour des concentrations en $\mathrm{TZ}$ de $5 \times 1 \mathrm{IO}^{-5}$ et $\mathrm{I} \times 1 \mathrm{IO}^{-4}$, les colonies apparaissent au bout de 48 à 72 heures.

Les différences dans la croissance en présence des différentes concentrations de $\mathrm{TZ}$ sont mises en évidence par la comparaison des diamètres moyens des colonies au bout de 4 jours d'incubation à $25^{\circ}$. Puisque la taille des colonies dépend du nombre de celles-ci par boite de Pétri, les mesures des diamètres sont faites dans des boites de Pétri ne contenant que 30 à 40 colonies.

Les résultats de ces mesures sont donnés par la courbe de la figure I 
dont le changement de pente à la concentration de $2,5 \times 10^{-5}$ indique le changement de la nature des colonies au delà et en deça de cette concentration de TZ. Les deux classes de colonies peuvent être différenciées par des tests plus rigoureux.

On sait que la réaction du Nadi, spécifique de l'indophénoloxydase, est négative chez les mutants " petite colonie " (Slonimski et Ephrussi, 1949) et positive chez la levure normale. Des échantillons de colonies ayant proliféré en présence de diverses concentrations en TZ sont prélevés et repiqués en milieu nutritif liquide. Après ro passages de 48 heures les cellules sont soumises au test du Nadi. Les résultats obtenus sont les suivants : toutes les colonies prélevées sur les milieux contenant de $\mathrm{I} \times \mathrm{IO}^{-4}$ à $2,5 \times 1 \mathrm{IO}^{-5} \mathrm{de} \mathrm{TZ}$ ont engendré des clones Nadi-négatifs. Par contre, tous les clones formés par des colonies prélevées sur des milieux à concentration en $\mathrm{TZ}$ inférieure à $\mathrm{I} \times \mathrm{IO}^{-5}$ sont Nadi-positifs.

L'examen. spectroscopique effectué sur les cultures dérivées des colonies ayant proliféré en présence de concentrations élevées en $\mathrm{TZ}\left(\mathrm{I} \times \mathrm{IO}^{-4}\right.$ à $\left.2,5 \times 10^{5}\right)$ met en évidence l'absence des bandes des cytochromes $a$ et $b$, caractéristique des mutants " petite colonie" (Slonimski et Ephrussi, 1949). Ces bandes sont au contraire présentes chez les levures prélevées sur des milieux dépourvus de TZ ou n'en contenant que de faibles concentrations $\left(\mathrm{I} \times \mathrm{IO}^{-6}\right.$ à $\left.\mathrm{I} \times \mathrm{IO}^{-5}\right)$.

En dehors des colonies normales observées sur les milieux contenant de o à $\mathrm{I} \times \mathrm{IO}^{-5} \mathrm{de} \mathrm{TZ}$, on y trouve une faible proportion de "petites colonie", omises lors de la mesure des diamètres. Le tableau I

TABLEAU I

Pourcentage de petites colonies formées en présence de diverses concentrations de TZ dars le milieu de culture

\begin{tabular}{|c|c|c|c|c|}
\hline \multirow{2}{*}{$\begin{array}{c}\text { Concentration du } \mathrm{TZ} \\
\text { (gr./l.) }\end{array}$} & \multicolumn{3}{|c|}{ Nombre de colonies } & \multirow{2}{*}{$\begin{array}{l}\text { Pourcentage des } \\
\text { petites colonies }\end{array}$} \\
\hline & Total & Normales & Petites & \\
\hline $\mathrm{I} \times \mathrm{IO}^{-4}$ & 2113 & $\ldots$ & 2113 & 10 \\
\hline $7,5 \times 10^{-5}$ & 2104 & $\begin{array}{l}\cdots \\
\cdots\end{array}$ & 2104 & xoo \\
\hline $5 \times 10^{-5}$ & 2049 & $\ldots$ & 2049 & 100 \\
\hline $2,5 \times 10^{-5}$ & 2036 & $\ldots$ & 2036 & 100 \\
\hline $1 \times 10^{-5}$ & 2295 & 2249 & 46 & 2,0 \\
\hline $5 \times 10^{-6}$ & I 993 & I 971 & 22 & $x, \mathbf{I}$ \\
\hline $1 \times 10^{-6}$ & 660 & 652 & 8 & 1,2 \\
\hline o & 2191 & 2172 & I9 & 0,9 \\
\hline
\end{tabular}

donne la fréquence des colonies mutantes, après étalement d'échantillons d'une même suspension de levure sur des milieux contenant diverses concentrations de TZ.*

On voit que les pourcentages des petites colonies trouvés pour

* Seul l'étalement sur $\mathrm{TZ}$ à $\mathrm{I} \times \mathrm{IO}^{-6}$ a été fait avec une suspension plus diluée. 
les concentrations en $\mathrm{TZ}$ de $\mathrm{I} \times \mathrm{IO}^{-6}$ et $5 \times \mathrm{IO}^{-6}$ sont sensiblement égaux au pourcentage de mutants spontanés des cultures témoins. Un léger accroissement de ce pourcentage est observé sur $\mathrm{TZ}$ à $\mathrm{I} \times \mathrm{IO}^{-5}$.* $^{*}$ Enfin, toutes les colonies formées en présence de $2,5 \times \mathrm{IO}^{-5}$ à $\mathrm{I} \times \mathrm{IO}^{-4}$ de $\mathrm{TZ}$ sont du type "petite colonie ".

Le fait que le nombre total de colonies obtenues à partir d'ensemencements égaux reste sensiblement le même quelle que soit la concentration en $\mathrm{TZ}$ suggère que l'accroissement du pourcentage des "petites colonies" n'est pas dû à la destruction élective des cellules normales par le TZ.

Suivant la concentration de TZ dans le milieu gélosé, les colonies de levure sont plus ou moins fortement colorées. Lorsque la concentration du TZ est comprise entre $1 \times 10^{-4}$ et $2,5 \times 10^{-5}$, la plus grande partie des colonies sont rouge pâle à rose pâle ; quelques colonies sont blanches. Lorsque la concentration est comprise entre $\mathrm{I} \times 1 \mathrm{IO}^{-5}$ et $\mathrm{I} \times 1 \mathrm{IO}^{-6}$, les colonies normales sont foncé rouge ou roses, les petites colonies roses ou blanches.

Il ressort de ce qui vient d'être dit que, pour certaines concentrations du milieu de culture en $\mathrm{TZ}$, il ne se forme que des colonies mutantes dont les cellules conservent leurs propriétés caractéristiques pendant de nombreuses générations.

Deux hypothèses peuvent rendre compte de ce fait :

(a) le TZ exerce un effet sélectif : dans un milieu contenant du TZ, les cellules mutées ont un avantage sélectif sur la forme normale. En conséquence, les mutants spontanés qui apparaissent dans toute culture de levure normale prennent le dessus en présence de TZ.

(b) le $\mathrm{TZ}$ a une action mutagène: il induit la mutation "petite colonie".

Se fondant sur les principes établis par Luria et Delbrück (1943), Ephrussi, Hottinguer et Leupold (inédit) ont mis au point une technique qui permet de trancher entre les deux hypothèses et de comparer les taux de mutation dans deux séries de cultures, en l'occurrence en présence et en absence de TZ.

Une goutte d'une culture de levure est placée dans la chambre à microdissection. Des cellules individuelles y sont prélevées et placées isolément soit dans des gouttelettes de milieu normal (témoins), soit dans des gouttelettes du même milieu contenant $5 \times 10^{-5}$ de $\mathrm{TZ}$. La chambre est incubée à $25^{\circ}$. Lorsque le nombre de cellules dans les clones formés par chacune des cellules isolées atteint Ioo à 200, les cultures sont retirées de la chambre à microdissection par aspiration dans une micro-pipette et étalées sur milieu gélosé normal en boites

* A cette concentration, le pourcentage des petites colonies est encore faible, mais toutes les grandes colonies sont "festonnées". D'apres Ephrussi et ses collaborateurs (Ephrussi, 1949; Ephrussi, L'Héritier et Hottinguer, 1949), une colonie festonnée, dissociée et rétalée, donne naissance à un mélange de grandes et de petites colonies. Les colonies festonnées sont donc probablement le résultat d'un équilibre entre cellules normales et cellules mutées. 
de Pétri. Au bout de 3 à 4 jours de croissance à $25^{\circ}$, le nombre de colonies de chaque type ("grandes" et " petites") est déterminé.

Le tableau 2 donne les résultats d'une expérience typique, et le tableau 3 un résumé des résultats de l'ensemble des expériences effectuées.

TABLEAU 2

Fréquence des petites colonies dans les clones formés par des cellules isolées en milieu normal ou contenant du TZ (résultats d'une cxpérience type)

\begin{tabular}{|c|c|c|c|c|c|c|c|c|c|}
\hline \multirow{2}{*}{\multicolumn{2}{|c|}{$\begin{array}{c}\text { Rapport } \\
\text { colonies/cellules }\end{array}$}} & \multicolumn{6}{|c|}{ Nombre de colonies } & \multirow{2}{*}{\multicolumn{2}{|c|}{$\begin{array}{l}\text { Pourcentage des } \\
\text { petites colonies }\end{array}$}} \\
\hline & & \multicolumn{2}{|c|}{ Total } & \multicolumn{2}{|c|}{ Normales } & \multicolumn{2}{|c|}{ Petites } & & \\
\hline $\mathrm{TZ}$ & Témoin & $\mathrm{TZ}$ & Témoin & $\mathrm{TZ}$ & Témoin & $\mathrm{TZ}$ & Témoin & $\mathrm{TZ}$ & Témoin \\
\hline 0,53 & $0,5^{8}$ & 46 & 47 & 34 & 46 & I 2 & $I$ & 26,1 & $2, I$ \\
\hline 0,54 & $0,4 I$ & $5^{2}$ & 42 & 43 & $4^{2}$ & 9 & 0 & 9,3 & $o$ \\
\hline 0,63 & $0,5 I$ & 74 & 54 & 57 & 54 & 17 & 0 & 22,9 & 0 \\
\hline 0,58 & 0,59 & 34 & $6 I$ & 0 & $6 I$ & 34 & 0 & 100 & 0 \\
\hline $0,5 \mathrm{I}$ & 0,55 & 62 & $5 I$ & 56 & $5^{I}$ & 6 & 0 & 9,7 & 0 \\
\hline 0,60 & 0,60 & $5^{8}$ & 70 & 38 & 70 & 20 & $o$ & 34,5 & 0 \\
\hline 0,69 & 0,57 & 74 & $5 I$ & 70 & $5^{I}$ & 4 & 0 & 5,4 & 0 \\
\hline 0,63 & 0,63 & 59 & 83 & 35 & 83 & 24 & $o$ & 40,7 & 0 \\
\hline 0,76 & 0,57 & 86 & $5^{6}$ & 55 & 56 & $3^{i}$ & $o$ & 36,0 & 0 \\
\hline 0,46 & 0,65 & 55 & 96 & 40 & 96 & I5 & $o$ & 27,3 & $o$ \\
\hline 0,50 & $0,5^{8}$ & 46 & 105 & 33 & 104 & 13 & $I$ & 28,3 & 0,9 \\
\hline
\end{tabular}

On constatera tout d'abord que le rapport entre le nombre de cellules dans un clone et le nombre de colonies formées après étalement de celui-ci sur milieu gélosé est le même $(0,69-0,70)$ pour les clones ayant proliféré en présence et en absence de $\mathrm{TZ}$. La valeur de ce

TABLEAU 3

Résumé de l'ensemble des expériences sur la fréquence des petites colonies dans les clones formés par des cellules isolées en milieu normal contenant du $T Z$

\begin{tabular}{|c|c|c|c|c|c|c|c|c|}
\hline Milieu & \multicolumn{2}{|c|}{ Nombre de } & $\begin{array}{c}\text { Rapport : } \\
\text { colonies/ } \\
\text { cellules }\end{array}$ & \multicolumn{2}{|c|}{$\begin{array}{c}\text { Nombre de } \\
\text { colonies }\end{array}$} & $\begin{array}{c}\text { Pourcentage } \\
\text { de petites } \\
\text { colonies }\end{array}$ & $\begin{array}{c}\text { Clones } \\
\text { sans } \\
\text { petites } \\
\text { colonies }\end{array}$ \\
\cline { 2 - 8 } & clones & cellules & colonies & & normales & petites & & \\
\hline TZ & 38 & 4634 & 3229 & 0,70 & 2450 & 779 & 24,1 & 2 \\
Normal & 43 & 6687 & 4612 & 0,69 & $45^{8}$ & 27 & 0,6 & 26 \\
\hline
\end{tabular}

rapport s'explique de la façon suivante : lorsqu'on compte le nombre de cellules dans un clone, on compte forcément un certain nombre de bourgeons qui ne se détachent pas de la cellule-mère lors de l'étalement. Ainsi, dans un certain nombre de cas, ce qu'on a compté comme 2 cellules ne donne naissance qu'à une seule colonie.

On constate d'autre part que le pourcentage des petites colonies 
est beaucoup plus élevé dans les clones ayant proliféré en présence de TZ (24, I p. cent.) que dans les clones témoins ( 0,6 p. cent.), et que la proportion de clones n'ayant formé aucune petite colonie est beaucoup plus faible dans la série $\mathrm{TZ}$ que dans la série témoin (tableau 3, dernière colonne).

Ces chiffres permettent de calculer les taux de mutation pour les deux séries de cultures, selon les principes indiqués par Luria et Delbrück (1943). Afin d'éviter le facteur sélection, on calcule les taux de mutation $a$ à partir du rapport : clones sans mutants/nombre total de clones, en utilisant l'équation :

$$
a=\frac{\ln 2 \times \ln \left(\mathrm{C}_{0} / \mathrm{C}\right)}{\mathrm{N}}
$$

où $\mathrm{C}_{0}$ est le nombre de clones sans mutants, $\mathrm{C}$ le nombre total de clones, et $\mathrm{N}$ le nombre moyen de colonies par boite de Pétri. Ge calcul fournit $\mathrm{a}=2,3 \times \mathrm{IO}^{-2}$ pour les cultures en $\mathrm{TZ}$ et $3,3 \times \mathrm{ro}^{-3}$ pour les témoins, et montre donc qu'à la concentration de $5 \times 10^{-5}$ le $T Z$ cause une élévation du taux de mutation de ro fois environ.

Notons cependant que la différence entre les taux de mutation dans les deux séries de cultures est probablement sous-estimée par ce calcul. Celui-ci est en effet fondé sur les chiffres des tableaux 2 et 3 qui, on se souvient, sont le résultat du classement des colonies d'après leur diamètre. Or, si l'on prélève des colonies ainsi classées " petites" et si on les soumet à une série de passages, suivis du test de la réaction du Nadi, on trouve que la très grande majorité des clones dérivés des colonies de taille réduite de la série témoin sont Nadi-positifs, tandis que tous les clones correspondants de la série TZ sont Nadinégatifs.

Des échantillons provenant de 14 petites colonies trouvées dans les cultures en $\mathrm{TZ}$ et de 14 petites colonies trouvées dans les cultures témoin ont été prélevés. Après Io passages de 48 heures en milieu liquide dépourvu de TZ, les cultures ont été soumises à la réaction du Nadi. Cette réaction s'est révélée négative pour toutes les cultures de la série $\mathrm{TZ}$ et positive pour celles de la série témoin.

La nature des "fausses petites" (" petites réversibles" d'Ephrussi, L'Heritier et Hottinguer, r949) étant inconnue, il serait raisonnable, du point de vue où nous nous plaçons dans ce travail, de ne considérer comme mutants véritables que ceux dans la descendance desquels on n'a pas observé de "réversion" vers la forme normale. Si l'on accepte cette manière de voir, il apparaît qu'en nous fondant sur le seul critère morphologique, nous avons, dans le calcul ci-dessus, considérablement surestimé la fréquence des mutations spontanées et sous-estimé la différence entre les taux de mutation dans les deux séries de clones, puisque les "fausses petites" sont particulièrement fréquentes dans la série témoin.*

* Le très petit nombre de déterminations ne nous permet de donner qu'une estimation maxima du taux de mutation spontáné fondée sur l'emploi du test du Nadi. Elle fournit la valeur $1,1 \times 10^{-3}$. Selon Ephrussi et ses collaborateurs, la valeur réelle est plus proche de $1,1 \times 10^{-4}$ (communication personnelle). 
Nous avons pensé qu'en utilisant des concentrations plus élevées de TZ il serait possible de donner une démonstration plus directe de l'action mutagène de cette substance par l'emploi de la technique d'Ephrussi et Hottinguer (1950). Une cellule (cellule-mère) provenant d'une culture normale de levure est placée à l'aide du micromanipulateur dans une gouttelette de milieu nutritif contenant du

TABLEAU 4

Résultats de l'étude de la descendance de 3 cellules proliférant en présence de $T Z$ et de 3 cellules témoins

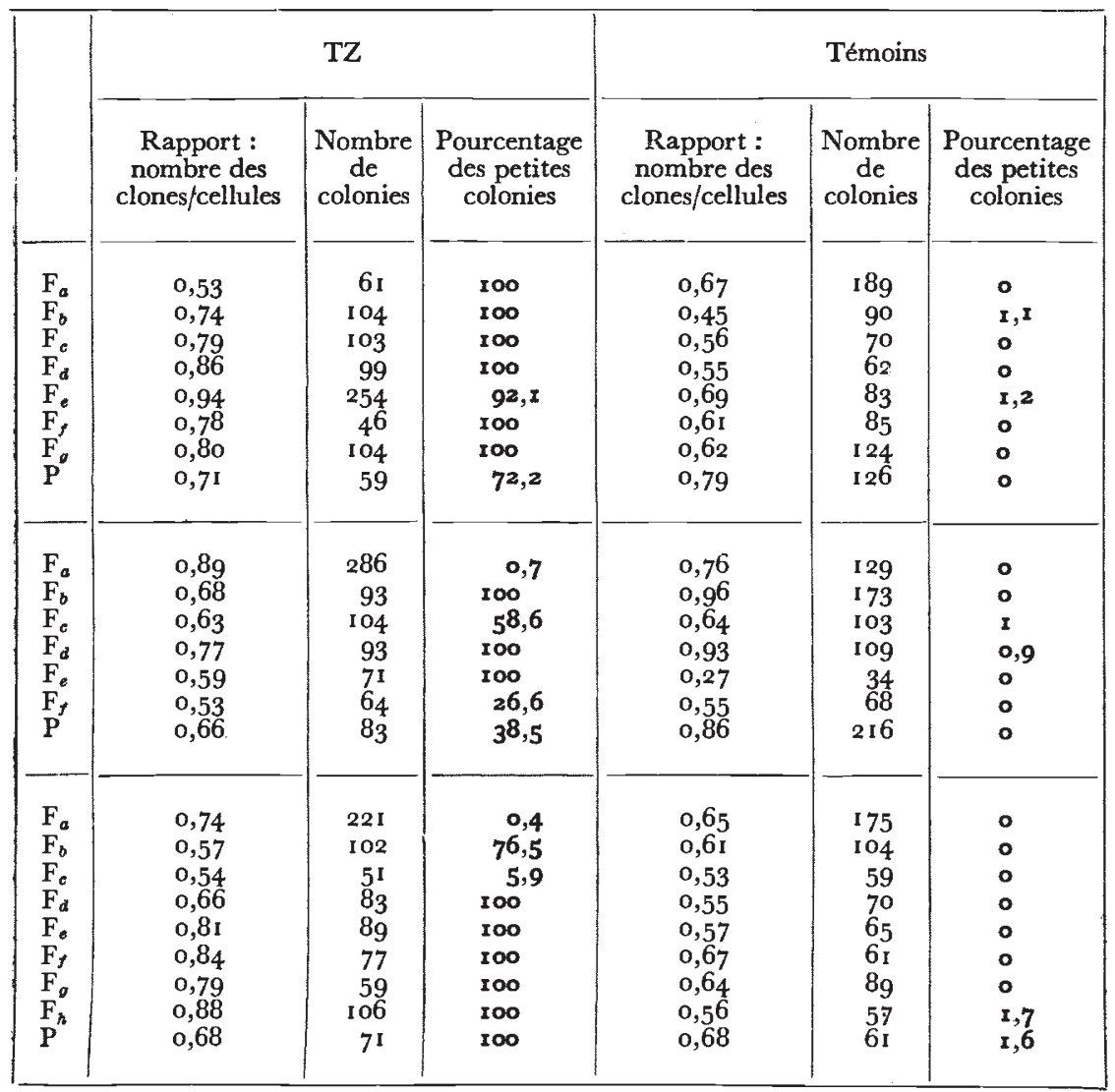

TZ. Le bourgeonnement de la cellule-mère $(\mathrm{P})$ est observé au microscope, et les cellules-filles $\left(\mathrm{F}_{a}, \mathrm{~F}_{b}, \mathrm{~F}_{c}\right.$, etc. $)$ sont séparées de la cellulemère au fur et à mesure de leur formation, et transportées chacune dans une gouttelette de milieu nutritif normal. A la fin de l'expérience, la cellule-mère elle-même est transportée dans une gouttelette de milieu normal. Des cellules isolées et traitées de la même façon dans du milieu dépourvu de $\mathrm{TZ}$ servent de témoin. Lorsque les cellules ainsi isolées ont formé des clones contenant de 100 à 200 cellules, ceux-ci sont retirés de la chambre à microdissection et étalés sur 
milieu gélosé normal en boites de Pétri. Au bout de 3 à 4 jours, on $\mathrm{y}$ dénombre les colonies de taille normale et réduite, et on prélève un certain nombre des dernières pour les soumettre, après une série de repiquages, à l'examen spectroscopique.

Le tableau 4 donne, à titre d'exemple, les résultats de 6 expériences, dont 3 ont été effectuées en présence de $\mathrm{TZ}$ à $2 \times \mathrm{ro}^{-4}, 3$ autres en milieu normal (témoins), et le tableau 5 résume l'ensemble des expériences réalisées avec cette concentration de $\mathrm{TZ}$ et des expériences témoins.

L'examen de ces tableaux permet de constater que

(I) Les clones témoins ne forment le plus souvent pas de petites colonies ou n'en forment qu'un petit pourcentage. Seulement dans 8 cas sur 48 le pourcentage des petites colonies a dépassé 2 per cent. (maximum observé : 22 per cent.).

\section{TABLEAU 5}

Résumé des résultats fournis par l'étude de la descendance des cellules isolées en milieu normal ou contenant $d u T Z$

\begin{tabular}{|c|c|c|c|c|}
\hline \multirow{2}{*}{ Milieu } & \multicolumn{3}{|c|}{ Nombre des cellules } & Nombre de clones mixtes \\
\cline { 2 - 5 } & $\mathrm{P}$ & $\mathrm{F}$ & F mutés & $\begin{array}{c}\text { Nontenant plus de 2 p. cent. } \\
\text { de "petites colonies" }\end{array}$ \\
\hline TZ & 7 & 45 & 18 & 17 \\
Normal & 6 & 48 & 0 & 8 \\
\hline
\end{tabular}

(2) Par contre, parmi les colonies formées par les clones de la série TZ, un pourcentage élevé sont de taille réduite. Sur 45 clones, I 7 ont formé plus de 2 per cent. de petites colonies et 18 autres ont formé uniquement des petites colonies, ce qui permet de conclure que les 18 cellules $\mathrm{F}$ qui sont à l'origine de ces clones étaient ellesmêmes mutées. Puisque aucun des 48 clones $F$ de la série témoin ne s'est comporté de façon semblable, il est clair que le $T Z$ exerce une action mutagène. Cette conclusion est encore confirmée par le fait que, dans la série $T Z$, les clones engendrés par les cellules-mères $(P)$ après leur transfert dans le milieu normal ont formé soit uniquement des petites colonies, soit un mélange de colonies des deux tailles. Dans un certain nombre de cas, le TZ fait donc muter les cellules-mères elles-mêmes, ou y induit un "état instable" qui se manifeste par la production fréquente de bourgeons mutés (Ephrussi et Hottinguer, I95I). La mutation des cellules-mères au cours de l'expérience n'a jamais été observée chez les témoins, et les rares cas où les clones témoins contiennent un pourcentage relativement élevé de petites colonies doit être attribué à l'intervention précoce, dans l'histoire du clone, d'une mutation spontanée. 
Les données fournies par les expériences qui viennent d'être décrites permettent de calculer les taux de mutation dans les séries $\mathrm{TZ}$ et témoins, suivant la formule

$$
\pi=\frac{n_{p}}{n_{g}+n_{p}}
$$

où $n_{g}$ est le nombre de grandes et $n_{p}$ le nombre de petites colonies formées par un certain nombre de cellules normales (Marcovich, 195I). Le calcul ne sera fondé, bien entendu, que sur celles des expériences en $\mathrm{TZ}$ où la cellule-mère elle-même n'a pas subi de mutation avant l'isolement du dernier bourgeon. D'autre part, on ne considèrera comme mutantes que les cellules-filles dont la descendance n'a formé que des petites colonies. Dans ces conditions, le calcul fournit, pour la série TZ, $\pi=0,32 . *$ Puisque dans la série témoin aucune cellule $\mathrm{F}$ mutante n'a été trouvée, on ne peut donner qu'une estimation de la valeur maxima de $\pi$ qui est $<0,02$.

Les expériences ci-dessus étant réalisées de faģon à exclure le facteur de sélection (la mortalité des cellules y est pratiquement nulle), les résultats obtenus établissent l'induction, par le TZ, des mutants à déficience respiratoire. On notera cependant qu'une analyse génétique serait nécessaire pour déterminer s'il s'agit ici de mutants cytoplasmiques ou géniques.

\section{RESUMÉ}

(I) En présence dans le milieu de culture de certaines concentrations de 2.3.5. triphényltétrazolium (TZ), les levures ne forment que des colonies de taille réduite qui présentent les mêmes caractéristiques morphologiques et physiologiques que les mutants à déficience respiratoire décrits par Ephrussi et ses collaborateurs.

(2) Le pouvoir mutagène du TZ a été établi par des expériences réalisées dans des chambres à microdissection, dans des conditions qui excluent la sélection, et les taux des mutations induites ont pu être calculés.

\section{SUMMARY}

(I) Baker's yeast, grown in the presence of certain concentrations of 2.3.5. triphenyltetrazolium chloride (TZ), produces dwarf colonies, the cells of which are morphologically and physiologically similar to the respiration deficient mutants described by Ephrussi et al.

(2) The mutagenic effect of $\mathrm{TZ}$ is established by experiments performed in the microdissection chamber, under conditions excluding selection. The induced mutation rate is determined.

Je tiens à remercier le Professeur B. Ephrussi de m'avoir suggéré le sujet de ce travail, ainsi que des conseils et des critiques qu'il a bien voulu me prodiguer.

* Ce calcul est fondé encore une fois sur les données fournies par l'observation des colonies. Le taux de mutation ainsi calculé doit cependant être très proche de la vérité puisque sur 32 petites colonies isolées dans ces expériences, $3^{\mathrm{I}}$ ont formé des clones dont l'examen spectroscopique, après 6 à 9 passages, a confirmé la nature mutante. 


\section{BIBLIOGRAPHIE}

EPHRUSSI, B. 1949. Action de l'acriflavine sur les levures. In Unités biologiques douées de continuité génétique. G.N.R.S., Paris.

EPHRUSSI, B., ET HOTTINGURR, H. 1950. Direct demonstration of the mutagenic action of euflavine on baker's yeast. Nature, $166,956$.

EPHRUSSI, B., ET hOTTINGUER, H. I95I. Cytoplasmic constituents of Heredity. On an unstable cell state in yeast. Cold Spring Harbor Symposia on quantitative Biology, 16, 75-85.

EPHRUSSI, B., HOTTINGURR, H., ET CHIMENES, A. M. I949. Action de l'acriflavine sur les levures. I. La mutation " petite colonie". Ann. Inst. Pasteur, 76, 35I-367.

EPHRUSSI, B., HOTTINGUER, H., ET TAVITTZK, J. I949. Action de l'acriflavine sur les levures. II. Etude génétique du mutant "petite colonie". Ann. Inst. Pasteur, 76, 419-450.

EPHRUSSI, B., L'HERTTIER, PH., ET hOTTINGUER, H. I949. Action de l'acriflavine sur les levures. VI. Analyse quantitative de la transformaton des populations. Ann. Inst. Pasteur, 77, 64-83.

LURIA, S. E., ET DELBRÜCK, M. I943. Mutations of bacteria from virus sensitivity to virus resistance. Genetics, $28,49 \mathrm{I}-5 \mathrm{I}$ I.

marcovich, H. I95I. Action de l'acriflavine sur les levures. VIII. Détermination du composant actif et étude de l'euflavine. Ann. Inst. Pasteur, 81, 452-468.

RAUT, c. I953. A cytochrome deficient mutant of Saccharomyces cerevisiz. Exp. Cell Research, 4, 295-305.

SLONIMSKr, P. P., ET EPHRUsst, B. 1949. Action de l'acriflavine sur les levures. V. Le systeme des cytochromes des mutants "petite colonie". Ann. Inst. Pasteur, 77, 47-63. 